\title{
History of domestication and spread of Aedes aegypti - A Review
}

\author{
Jeffrey R Powell ${ }^{1 /+}$, Walter J Tabachnick ${ }^{2}$ \\ ${ }^{1}$ Department of Ecology and Evolutionary Biology, Yale University, New Haven, CT, USA \\ ${ }^{2}$ Florida Medical Entomology Laboratory, Institute of Food and Agricultural Sciences, University of Florida, Vero Beach, FL, USA
}

\begin{abstract}
The adaptation of insect vectors of human diseases to breed in human habitats (domestication) is one of the most important phenomena in medical entomology. Considerable data are available on the vector mosquito Aedes aegypti in this regard and here we integrate the available information including genetics, behaviour, morphology, ecology and biogeography of the mosquito, with human history. We emphasise the tremendous amount of variation possessed by Ae. aegypti for virtually all traits considered. Typological thinking needs to be abandoned to reach a realistic and comprehensive understanding of this important vector of yellow fever, dengue and Chikungunya.
\end{abstract}

Key words: Aedes aegypti - history biogeography - domestication - arboviruses

"When to this is added the fact that the more closely a mosquito is associated with man, the more is it the subject of prejudice and misconception, it follows that the prevailing conception of Ae. aegypti in the minds of the general run of entomologists may well be more remote from reality than in the case of most other mosquitoes."

Mattingly (1957)

As humans have grown in numbers and occupancy of the Earth, their habitats have encroached on the native habitats of many species. One outcome is extinction of the invaded species, another is evolution of "domestication" or commensalism, the breeding in human-occupied territory. When this occurs for insects that require a vertebrate source of blood, the results can be disastrous. These blood-requiring insects most often evolve a preference for the most available and stable blood source: humans. Many major insect vectors of human diseases have undergone this domestication process and now breed in close proximity with humans and take human blood meals.

One consequence of this switch in taking blood meals from non-human animals, zoophagy, to anthropophagy is that humans are challenged with infectious diseases once confined to animals. Humans are a relatively recent member of the Earth's biota having arisen less than 10 million years ago. Blood-feeding insects have been around for hundreds of millions of years. Thus it is safe to assume that the various infectious agents transmitted by insects have a long history with non-human animals and that their infection of humans is a recently derived phenomenon. Many human vector-borne pathogens to-

doi: 10.1590/0074-0276130395

Financial support: NIH (RO1 AI046018, RO1 AI101112) (to JRP)

+ Corresponding author: jeffrey.powell@yale.edu

Received 9 August 2013

Accepted 15 October 2013 day also infect animals; those that do not, have close relatives infecting animals.

A second consequence of commensalism is the potential for the spread of vectors outside their previous "native" range, i.e., becoming an invasive species. Because of all species, humans occupy the widest range of habitats on Earth, once a species evolves the ability to co-exist with humans they will likely be spread by the great mobility of humans. Lounibos (2002) provides an excellent synopsis of the importance of invasiveness in insect vectors.

So, from a public health perspective, the evolution of vector domestication is an extremely important phenomenon, yet has not received the close study it would seem to warrant. Here we focus on Aedes aegypti, a widespread species of mosquito that has both domestic populations as well as the ancestral type that still extant in sub-Saharan Africa. This will be done in the context of ongoing work on the evolutionary genetics of this species. Behavioural changes associated with domestication are particularly important and are emphasised here. Because insect behaviour genetics was the focus of Alexandre Peixoto's brilliant, but all too short, career, this theme is a fitting tribute to his memory.

History of Ae. aegypti - While the official common name for this species is the "yellow fever mosquito", today it is of most public health concern as the major vector of dengue fever. Due to an effective vaccine, yellow fever is of less concern worldwide, although cases still occur (Barrett \& Higgs 2007). Generally, Ae. aegypti is important in spreading viral diseases such as yellow fever, dengue fever and Chikungunya.

Tabachnick (1991) reviewed many of the ideas about the history of Ae. aegypti's distribution throughout the world given the information at that time. It is almost certain that the ancestor of the domestic form of $A e$. aegypti lived in sub-Saharan Africa. The larval habitat was likely tree holes and non-human animals provided blood meals. Today, this ancestral form still exists in forests and vegetated ecotones in sub-Saharan Africa (Lounibos 
1981) and is called by a subspecies name, formosus. In addition to laying eggs in tree holes and preferring nonhuman blood, morphologically this form is much darker than the form adapted to human habitats, although this morphology based on scaling patterns is quite variable (McClelland 1974) and, as will be clear later, is decoupled from the behavioural traits associated with urban vs. sylvan breeding in different parts of the world.

Two scenarios have been put forward for the origin of the light-coloured domestic subspecies, Ae. aegypti aegypti (for ease of communication, from here on we refer to forest-breeding populations in sub-Saharan Africa as the classically defined formosus subspecies as Aaf and the light coloured populations outside of outside Africa as Aaa. However, as will become clear, this simple dichotomy masks the true complexity of the species). Almost certainly Ae. aegypti came to the New World on ships where conditions were such as to select for a domestic type. The two scenarios differ in whether the species had already become domesticated prior to spread (i.e., preadapted to human transport) or became domesticated in response to transport. The species was likely once more widespread including in forested northern Africa before the formation of the Sahara Desert. As the north part of the continent dried over the last 4,000-6,000 years forming the Sahara (Kropelin et al. 2008), populations along the northern coast and around the Mediterranean would have become isolated from the sylvan form south of the Sahara. As the drying continued, the only reliable water sources for northern populations were those found in human settlements. Interestingly, a third subspecies, Aedes aegypti queenslandensis, was described as a particularly light coloured form found in the Mediterranean Basin (Mattingly 1967). As Ae. aegypti has been eradicated in the Mediterranean Basin, it is not clear if queenslandensis still exists although we do know it was certainly a domestic form.

Whether the domestication event preceded or occurred simultaneous with its introduction into the New World, Ae. aegypti arrived soon after Europeans first arrived. Yellow fever was known in sub-Saharan Africa much before 1400, but was not known in the New World prior to European arrival. The first confirmed outbreak of yellow fever in the New World occurred in the Yucatan, in 1648 (McNeill 1976), although yellow fever may have been in Haiti as early as 1495 (CloudsleyThompson 1976).

The early trade between the Old and New Worlds has been described as "triangular" (Murphy 1972). Ships from Portugal and Spain sailed to West Africa to acquire slaves, brought them to the New World where they were exchanged for goods that were brought back to Portugal and Spain. Whether the ships acquired Ae. aegypti in West Africa or already had the domestic form when they originated in Europe is not clear. Aaa as it occurs in the New World is not known in West Africa today, except perhaps as a reintroduction into ports (Brown et al. 2011).

Evidence from DNA sequencing and large-scale single nucleotide polymorphisms (SNP) analyses indicate that following introduction into the New World the species likely spread westward across the Pacific into Asia and Australia (Figure). Populations in the New World are derived directly from African populations, while Asia/Australian populations are derived from New

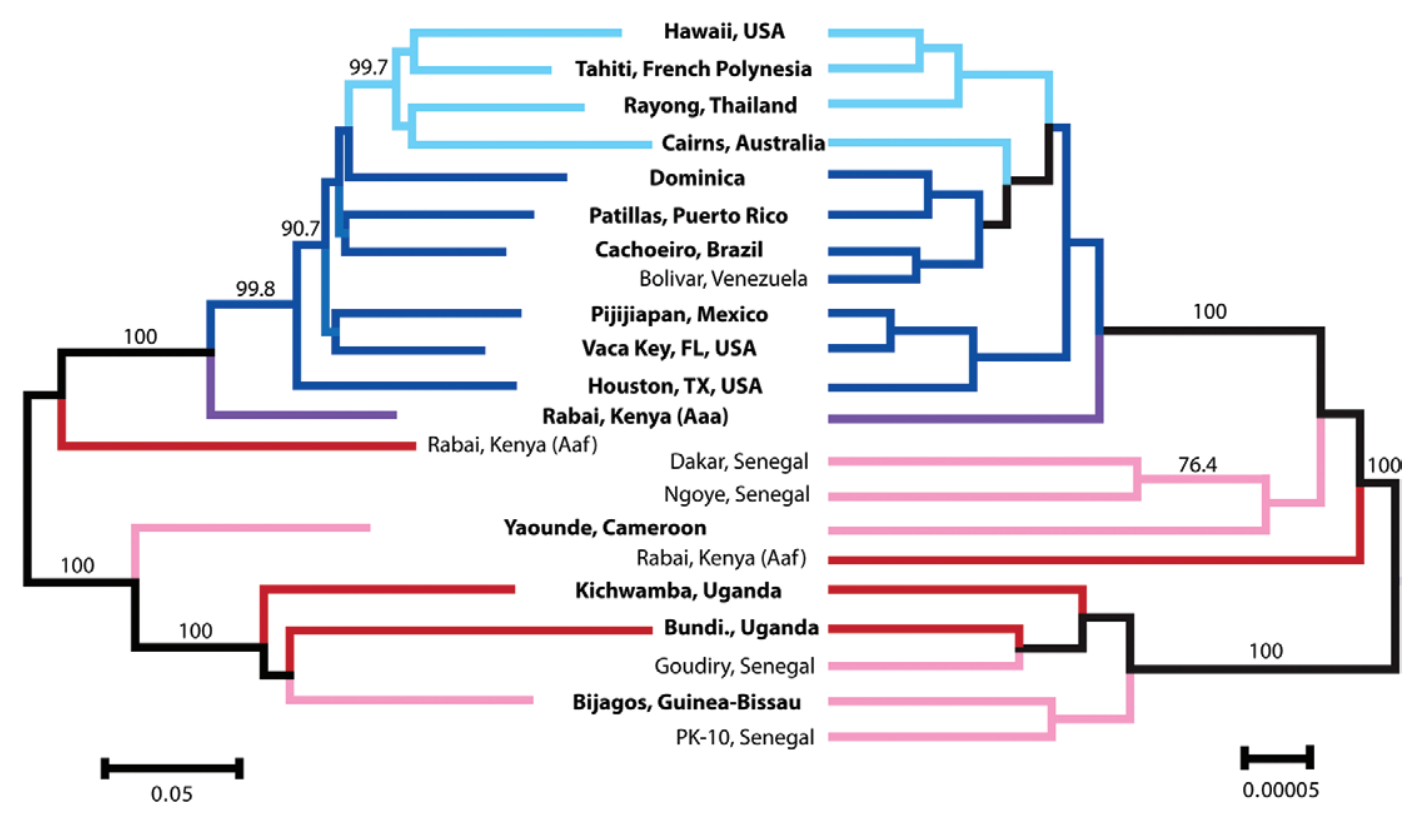

Evolutionary history of Aedes aegypti from single nucleotide polymorphisms (SNPs) and sequenced nuclear genes. Bootstrapped neighbourjoining network based on population pairwise chord-distances from 1,504 SNPs (left). Bayesian population tree based on phased DNA sequences of genes listed in Table. Node support over $75 \%$ is shown on relevant branches. East African populations are shaded in red, West and Central African populations in pink, the Rabai domestic (called Aaa here) population in purple, New World populations in dark blue and Asia-Pacific populations in light blue. Rooting was inferred from DNA sequences of three nuclear genes from Aedes mascarensis (Brown et al. 2013). 
World populations. A second piece of genetic information favouring Africa to New World to Asia/Australia is the level of genetic variation. Table summarises the information. As would be expected by two successive founding events, the amount of genetic variation decreases from Africa to the New World and then again from the New World to Asia/Australia. The westward expansion from the New World to Asia is surprising given that an eastern migration from East Africa to Asia might be expected based on geography and the historic intensive trade between India and East Africa. We have not yet seen genetic evidence of this, although it must be noted that our sampling in Asia is sparse especially with regard to the Indian Subcontinent. However, recent analyses of samples from Saudi Arabia are placed with other Asian populations (A Gloria-Soria \& JR Powell, unpublished observations). Data from allozymes indicated that samples from India were not genetically different from those from Indonesia and Taiwan (Wallis et al. 1983). So as far as we know the colonisation out of Africa was unidirectional, westward (although see below). The timing of Ae. aegypti colonisation of Asia is likely the late XIX century when dengue fever was first reported and, importantly, in urban settings due to the arrival of the only urban dengue vector, Ae. aegypti (Smith 1956). [The historic absence of yellow fever in Asia, despite the presence of Ae. aegypti, remains one of medical entomology's great mysteries. Several explanations for this have been proposed (Tabachnick 2013)].

Why might East Africa not be a source for nearby Asia regions? Except for the unusual situation in east Africa highlighted by Rabai, Kenya (discussed next), a domestic form of Ae. aegypti capable of passive transport by humans may not have existed in East Africa. Yellow fever was unknown or very rare in East Africa until recently (Mutebi \& Barrett 2002) and what epidemics occurred have been sylvan and transmitted by Aedes species other than Ae. aegypti (Saunders et al. 1998). In fact, Mutebi and Barrrett (2002) state that “... in West Africa, Ae. aegypti is responsible for urban YF outbreaks, whereas in East Africa, Ae. aegypti has never been incriminated in the transmission of YF virus". Thus epidemiologic data indicate East African aegypti are very different from West African and either are not sufficiently associated with humans and anthropophilic and/or not as competent to transmit yellow fever. The fact that East African aegypti are not favourable vectors of human diseases indicates limited adaptation to human environments, perhaps precluding them from surviving aboard ships for long periods as would be required for East Africa to Asia migration on ships.

While this origin of present day Asian Ae. aegypti by colonization from the New World is consistent with the current genetic data, an alternative scenario based on historical considerations was proposed by Tabachnick (1991). The origin of domestic Ae aegypti is posited to have occurred in North Africa as described; whether this initial form was more similar to the description of Aaa or subspecies queenslandensis is not known. The introduction of domestic aegypti to West Africa may have occurred via human trade at a time when introgression with sylvan aegypti occurred there, resulting in the domestic populations and morphology now observed in West Africa. The introduction of domestic aegypti into East Africa may have occurred much later, including into the Rabai region, where domesticity allowed it to remain sympatric with sylvan forms in this particular environment. Therefore domestic aegypti in Asia, now corresponding to Aaa elsewhere outside of Africa, would be a later arrival since it was a recent arrival to East Africa. This is also consistent with Smith's (1956) observation that Ae. aegypti likely arrived in Asia in the latter half of the XIX century since urban dengue epidemics were unknown until then, until the arrival of the only urban vector, Ae. aegypti. Figure powerful evidence arguing against the likelihood of East Africa Rabai region as the origin for Asian Ae. aegypti. A more direct route might have occurred to Asia from the North African ancestral Ae. aegypti with the opening of the Suez Canal and the accompanying increase in ship trade to the Indian subcontinent (Tabachnick 1991). This is consistent with the introduction of $A e$. aegypti to Asia in the latter part of the XIX century and consistent with what we know about human trade and migration that might support Ae. aegypti migration. Further studies will be needed to resolve these issues.

Perhaps most remarkably, the domestic form of $\mathrm{Ae}$. aegypti that now exists outside Africa throughout the tropical and sub-tropical world is a monophyletic group (Figure) (Brown et al. 2013). The implication is that the ancestral domestication event leading to the initial domestication of Ae. aegypti occurred once and all populations outside Africa are descended from this single lineage.

TABLE

Genetic variation statistics for four nuclear genes totalling approximately $2,500 \mathrm{bp}$ of sequence

\begin{tabular}{|c|c|c|c|c|c|c|c|c|}
\hline & \multicolumn{2}{|c|}{ apoLp-2 } & \multicolumn{2}{|c|}{ СYР9J2 } & \multicolumn{2}{|c|}{ SDR } & \multicolumn{2}{|c|}{ DVRF1 } \\
\hline & Hd & $\pi$ & Hd & $\pi$ & $\mathrm{Hd}$ & $\pi$ & $\mathrm{Hd}$ & $\pi$ \\
\hline Africa & 0.7152 & 0.0086 & 0.7908 & 0.0224 & 0.7638 & 0.0060 & 0.8871 & 0.0193 \\
\hline New World & 0.6406 & 0.0038 & 0.5150 & 0.0190 & 0.5700 & 0.0039 & 0.4949 & 0.0123 \\
\hline Asia-Pacific & 0.4843 & 0.0021 & 0.4158 & 0.0130 & 0.4343 & 0.0019 & 0.3750 & 0.0127 \\
\hline
\end{tabular}

genes with VectorBase accessions are apolipophorin (009955), cytochrome P450 (006805), short-chain degydrogenease/redutase (010137), dengue virus receptor 1 (008492) (Brown et al. 2013). Hd: haplotype diversity; Pi: per nucleotide site heterozygosity. 
Sympatry of domestic and sylvan Ae. aegypti - A possible exception to the general statements made above occurs along the east coast of Africa (Teesdale 1955, van Someren et al. 1958, McClelland 1973) best studied in the Rabai District of Kenya. Here both a domestic, light coloured form more or less (see later) corresponding to Aaa breeds in stored water in villages. Just a few hundred meters away, a form fitting the classical description of Aaf exist in the vegetated ecotones. These two forms have remained genetically distinct from one another over a period of at least 30 years (Tabachnick et al. 1979, Brown et al. 2011) and likely longer (Mattingly 1957). There are no reproductive barriers between the forms with hybrids and backcrosses perfectly fertile and, at least in the laboratory, they randomly mate with one another (Moore 1979). That these forms are truly sympatric is confirmed by finding the forest form in the huts at certain times of the year ( Trpis \& Hausermann 1978, Lounibos 2003). In addition to morphology and larval site differences, these two forms from Rabai display distinct differences in choice of host for blood meals, the indoor type preferring humans and the sylvan form non-human mammals (McClelland \& Weitz 1963, L McBride, unpublished observations).

Another trait of interest observed between the two forms from Rabai is oviposition preferences. Ae. aegypti females lay eggs just above the water line of natural pools (e.g., tree holes) or water in human-generated containers (e.g., flower pots, bird baths, discarded tires). The eggs remain dormant until flooded with water. Presumably this oviposition behaviour was adapted to natural conditions where water (rain) is unpredictable. If a pool is drying, eggs remain dormant; if rain is plentiful water rises to flood the eggs, they hatch and are more likely to have water long enough to undergo development. Lorimer et al. (1976) showed that the Rabai indoor form preferred clay surfaces such as the stored water jars in Rabai huts which is not the case with Aaa outside Africa. Evidently the oviposition cues are tactile in this case as opposed to the usually assumed olfactory (Lorimer et al. 1976, Lounibos 2003). Another unusual trait of East African indoor populations of Ae. aegypti is that it is that larval development is dependent upon permanent stored water. Indeed McClelland (1973) considered that the temporal stability of larval sites is more significant than natural (e.g. tree holes) vs. manmade containers because both are intermittently flooded by rain.

As can be seen in Figure and further documented in Brown et al. (2011, 2013), the domestic form found in Rabai, while morphologically and behaviourally (host choice) Aaa, is genetically distinct from other Aaa. What is the origin of this unique indoor form Ae. aegypti in Rabai? The phylogeny in Figure suggests this is an old lineage not closely related to other Aaa in the New World. Is this a surviving remnant of the Ae. aegypti queenslandensis described by Mattingly (1957) and once widespread around the Mediterranean? Indoor-breeding Ae. aegypti in coastal Kenya had been described as queenslandensis by Mattingly (1957). This subspecies was dependent upon stored water in human structures and its demise in the Mediterranean Basin coincided with the introduction of indoor plumbing in the early XX century (Curtin 1967,
Holstein 1967). As noted above the dependence on permanently stored indoor water persists in present day East African indoor populations (McClelland 1973).

Oviposition and "reversion" - As emphasised, the spread of Ae. aegypti out of Africa required the species to adapt to human environments with larval development in human-generated containers. Obviously this required a change in oviposition behaviour of ancestral sylvan females to first, enter human disturbed, even urban, environments and, second, to oviposit on metal, clay, rubber, etc., all of which would have been absent in its ancestral habitat. The adaptation for oviposition preference may have been part of the overall evolution of domesticity that likely occurred in North Africa when ancestral sylvan Aaf became isolated from sub-Saharan Africa due to the Sahara Desert (Tabachnick 1991). In general oviposition choice in mosquitoes is largely due to volatiles produced by the microorganisms in the larval water (although see exception mentioned earlier). Thus, as long as appropriate volatiles are produced by a standing pool of water, an opportunistic species like Ae. aegypti may oviposit there.

This is supported by situations where this domestic form outside Africa has reverted to developing in natural water. This has occurred mostly on islands or other isolated sites. Chadee et al. (1998) report 12 types of natural habitats where Aaa can be found in Jamaica, Puerto Rico and Trinidad including rock holes, tree holes, leaf axils, bamboo joints and coconut shells. Larvae developing in rock holes has been documented on the east coast of Africa (Trpis 1972) and in Anguilla (Wallis \& Tabachnick 1990). Aaa has been observed ovipositing in tree holes in New Orleans [cited in Wallis and Tabachnick (1990)].

In the case of rock hole larval sites on Anguilla, allozyme gene frequency differences were found in Anguilla between populations breeding in human-generated containers and rock hole Aaa a few kilometres apart (Wallis \& Tabachnick 1990) and the mosquitoes in the two habitats were also significantly differentiated with regard to development time and insecticide resistance (Tabachnick 1993). No difference was found in oviposition preferences.

[While not a natural site, as further evidence of the flexibility of Aaa on islands, larvae are found in septic tanks in Puerto Rico (Barrera et al. 2008), a niche more common for Culex pipiens and other mosquito species. No genetic differences were observed between surface and septic tank Aaa in Puerto Rico (Somers et al. 2011)]

This demonstrates that the species has remained adaptively flexible, maintains significant genetic variation for different life history traits and that breeding in human-generated containers is not a fixed trait outside of Africa. Aaa remains opportunistic, capable of rapidly responding to changes in environments. In the case of larval breeding sites, relatively few species of mosquitoes occur on islands, so not all potential mosquito larval niches are filled. In such cases, the invasive Aaa initially introduced into domestic habitats, spills over to occupy the empty natural niches. This is not in any sense a true "reversion" to the ancestral sylvan type; rather these are simply feral populations of what are genetically Aaa. 
Genetics of morphology - While morphology, in particular the colour of scales on abdominal tergites and background cuticle coloration, were important in differentiating the classical Aaa and Aaf, the detailed work of McClelland (1974) initially called such a simple dichotomy into question. He demonstrated that scaling patterns are highly variable within populations (as well as between). Many of the patterns have close resemblance to single gene Mendelian mutations known for this species (Munstermann 1993). In this regard the observations of Verna and Munstermann (2011) are instructive. Morphologically exceptional specimens were collected from a bucket on the island of Antigua that included a remarkably gold form. "The Antigua variants demonstrated morphology comparable to previously described mutations..." (Verna \& Munstermann 2011).

Thus the evidence is that scale pattern is a genetically highly variable character within and between populations of Ae. aegypti occupying various ecological niches; many of these patterns are due to variation at simple single Mendelian genes and not some complex of interacting genes that would take longer to evolve. Genetic relatedness as indicated by multiple molecular markers such as allozymes (Wallis et al. 1983), microsatellites (Brown et al. 2011) and 1,504 SNPs (Brown et al. 2013) often do not coincide with morphological similarities.

The conclusion is that variation in colouring exists within and between Ae. aegypti populations and only in some instances, in specific regions of the world, is that variation also indicative of the behavioural traits that lead to differences in adaptations. To our knowledge, no one has suggested an adaptive explanation for scaling colour variation in Ae. aegypti.

West Africa - The most dynamic situation with regard to domestication of Ae. aegypti is occurring in West Africa. Ae. aegypti have begun to breed in domestic habitats and it is clear that this is an independent evolutionary domestication from that leading to the spread of Aaa out of Africa. Domestic-breeding populations in West Africa have their closest relatives in sylvan populations in the same vicinity (Paupy et al. 2008, 2010, Brown et al. 2011) and are not closely related to Aaa outside Africa. While some workers describe domestic populations in West Africa as Aaa based on the presence of pale scales on the first abdominal tergite (Huber et al. 2008), its overall morphology especially cuticle colour is much darker than Aaa outside Africa and is more similar to sub-Saharan Aaf. The situation in Senegal is more complicated as there is evidence that Aaa from outside Africa has migrated back to Senegal (Brown et al. 2011) and thus domestic forms in Senegal exhibit some characteristics similar to Aaa outside Africa (Sylla et al. 2009).

The independent domestication occurring in West Africa opens the exciting possibility of studying the dynamics and genetics of this important event. This is almost certainly quite recent coinciding with expansion of human habitats and cities in West Africa and there are multiple independent incidents of sylvan populations moving into cities.
Epidemiology - In addition to all the traits so far discussed which are of importance from an evolutionary and ecological perspective, there is also considerable genetic variation in traits of importance for public health within and among populations of Ae. aegypti, in particular for their ability to transmit arboviruses, yellow fever and dengue in particular [reviewed in Black IV et al. (2002)]. Generally, populations described as Aaf have lower competence to transmit both dengue and yellow fever viruses than populations of Aaa. This brings up the intriguing possibility that the domestication process of mosquitoes has been accompanied by increase competence to transmit human viruses. Tabachnick (2013) posited out that vector competence is likely the result of the effects of adaptations for other functions not having anything to do with vector competence. In this view the adaptations accompanying domestication, whatever they are, have side effects that result in greater competence of Aaa, for example, to transmit yellow fever and dengue viruses.

Another reason for the correlation between the competence of domestic mosquitoes for human virus transmission could be adaptation of the virus to the mosquito. Once a mosquito like Ae. aegypti evolves to use humans as blood meals, there would be pressure on human arboviruses to adapt to this species of mosquito for transmission, in particular to the particular mosquito genotypes feeding on humans. It is clear with the related mosquito Aedes albopictus, that the arbovirus Chikungunya rapidly evolved to a new mosquito host (Tsetsarkin et al. 2011). So when an ancestrally zoophilic mosquito evolves anthropophily and introduces new viruses, the virus evolves to be efficiently transmitted through the human hosts and those mosquitoes feeding preferentially on humans. Others (Moncayo et al. 2004, Vasilakis et al. 2011) have also emphasised the importance of viral genotype in emergence of dengue. Evidently, the longer the evolutionary history of association of a mosquito with a virus, the more efficient the virus replicates in the arthropod host (Moncayo et al. 2004).

Variation abounds - The above emphasises just how much variation exists within the single species $A e$. aegypti. This is not unique to this vector as similar studies of insect vectors of disease have almost always revealed comparable variation (Tabachnick 2013). In the case of Ae. aegypti, one might make a partial list of such variable traits: (i) colour and pattern of scaling, (ii) host choice for blood meal, (iii) oviposition choice, (iv) larval sites, (v) egg dormancy, (vi) development time and (vii) competence to vector viruses.

These traits have both genetic and environmental components. The discussions argue that, at least to a large degree, these traits vary independently and thus are not always concordant. Considering this, it quickly becomes apparent that any attempt at categorising this species into two or three "subspecies" or other taxonomic unit is folly. While the classical definitions and designations of Ae. aegypti aegypti, Aedes aegypti formosus and Ae. aegypti queenslandensis were useful at one time and may sometimes still be useful in efficient commu- 
nication, developments in our understanding of the genetics and behaviour of this species have revealed the extent that this is a gross oversimplification of the true situation and represents typological thinking, discarded by most modern biologists. While we base this conclusion on recent genetic findings, an insightful early $A e$. aegypti expert, McClelland (1967) wrote: “...despite the population differences, Ae. aegypti cannot be split into definite infraspecific entities. In conclusion, Ae. aegypti may best be interpreted as a polymorphic rather than a polytypic species.". In the 45 years since, this advice has often been ignored, even in recent times.

\section{ACKNOWLEDGEMENTS}

Alexandre Afranio Peixoto was the academic grandchild of one of the authors (JRP, the PhD mentor to Louis Bernard Klaczko). JRP had the privilege of introducing Alexandre Afranio Peixoto as an academic "grandson" at an international meeting in Greece in 2011; Alexandre Afranio Peixoto responded with a big smile. To Julia E Brown and many other colleagues too numerous to list, for contributing to collection of more recent data from the Powell lab, and to Leon Lounibos, for making numerous insightful and detailed suggestions that greatly improved this paper.

\section{REFERENCES}

Barrera R, Amador M, Diaz A, Smith J, Munoz-Jordan JL, Rosario Y 2008. Unusual productivity septic tanks and its implications for dengue control. Med Vet Entomol 22: 62-69.

Barrett AD, Higgs S 2007. Yellow fever: a disease that has yet to be conquered. Ann Rev Entomol 52: 209-229.

Black IV WC, Bennett KE, Gorrochótegui-Escalante N, BarillasMury CV, Fernández-Salas I, Muñoz ML, Farfán-Alé JA, Olson KE, Beaty BJ 2002. Flavivirus susceptibility in Aedes aegypti: a review. Arch Med Res 33: 379-388.

Brown JE, Evans B, Zheng W, Abas V, Barrera-Martinez L, Egizi A, Zhao H, Caccone A, Powell JR 2013. Human impacts have shaped historical and recent evolution in Aedes aegypti, the dengue and yellow fever mosquito. Evolution doi: 10.1111/evo.12281.

Brown JE, McBride CS, Johnson P, Ritchie S, Paupy C, Bossin H, Fernandez-Salas I, Sylla M, Ponlawat A, Cornel AJ, Black WC, Gorrochotegui-Escalante N, Urdaneta-Marquez L, Slotman M, Walker C, Murray K, Powell JR 2011. Worldwide patterns of genetic differentiation imply multiple "domestications" of Aedes aegypti, a major vector of human diseases. Proc Biol Sci 278: 2446-2454.

Chadee DD, Ward RA, Novak RJ 1998. Natural habitats of Aedes aegypti in the Caribbean - a review. J Am Mosq Control Assoc 14: 5-11.

Cloudsley-Thompson JL 1976. Insects and history, St Martins Press, New York, 242 pp.

Curtin TJ 1967. Status of Aedes aegypti in the eastern Mediterranean. $J$ Med Entomol 4: 48-50.

Holstein M 1967. Dynamics of Aedes aegypti distribution, density and prevalence in the Mediteranean area. Bull World Health Organ 36: 541-543.

Huber K, Ba Y, Dia I, Mathiot C, Sall AA, Diallo M 2008. Aedes aegypti in Senegal: genetic diversity and genetic structure of domestic and sylvatic populations. Am J Trop Med Hyg 79: 218-229.

Kropelin S, Vershuren D, Lezine AM, Eggermont H, Cocquyt C, Francus P, Cazet JP, Fagot M, Rumes B, Russell JM, Darius F,
Conley DJ, Shuster M, von Suchodoletz H, Engstrom DR 2008. Climate-driven ecosystem succession in the Sahara: the past 6,000 years. Science 320: 7650768 .

Lorimer N, Lounibos LP, Petersen JL 1976. Field trials with a translocation homozygote in Aedes aegypti for population replacement. J Econ Entomol 69: 405-409.

Lounibos LP 1981. Habitat segregation among African treehole mosquitoes. Ecol Entomol 6: 129-154.

Lounibos LP 2002. Invasions by insect vectors of human disease. Ann Rev Entomol 47: 233-266.

Lounibos LP 2003. Genetic control trials and the ecology of Aedes aegypti at the Kenyan coast. In W Takken, WT Scott (eds.), Ecological aspects for application of genetically modified mosquitoes, Kluwer Academic, Dordrecht, p. 33-43.

Mattingly PF 1957. Genetical aspects of the Aedes aegypti problem: I Taxonomy and bionomics. Ann Trop Med Parasitol 51: 392-408.

Mattingly PF 1967. Taxonomy of Aedes aegypti and related species. Bull World Health Organ 36: 552-554.

McClelland GAH 1967. Speciation and evolution in Aedes. In JW Wright, R Pal (eds.), Genetics of insect vectors of disease, Elsevier, Amsterdam, p. 277-311.

McClelland GAH 1973. Some man-made mosquito problems in Africa and prospects for their rational solution. Proc Tall Timbers Conf Ecol Anim Control Hab Manage 5: 27-41.

McClelland GAH 1974. A worldwide survey of variation in scale pattern of the abdominal tergum of Aedes aegypti (L.) (Diptera: Culicidae). Trans $R$ Ent Soc Lond 126: 239-259.

McClelland GAH, Weitz B 1963. Serological identification of the natural hosts of Aedes aegypti (L.) (Diptera: Culicidae) caught resting in vegetation in Kenya and Uganda. Ann Trop Med Parasitol 57: 214-224.

McNeill WH 1976. Plagues and peoples, Knopf Doubleday Publishing Group, Garden City, 368 pp.

Moncayo AC, Fernandez Z, Ortiz D, Diallo M, Sall A, Hartman S, Davis CT, Coffey L, Mathiot CC, Tesh RB, Weaver SC 2004. Dengue emergence and adaptation to peridomestic mosquitoes. Emerg Infect Dis 10: 1790-1796.

Moore DF 1979. Hybridization and mating behavior in Aedes aegypti (Diptera: Culicidae). J Med Entomol 16: 223-226.

Munstermann LE 1993. Gene map of the yellow fever mosquito, Aedes (Stegomyia) aegypti $(2 \mathrm{~N}=6)$. In SJ O'Brien (ed.), Genetic maps: locus maps of complex genomes, Vol. 6, Cold Spring Harbor, New York, p. 3264-3268.

Murphy EJ 1972. History of African civilization, Dell Publishing, New York, 240 pp.

Mutebi JP, Barrett ADT 2002. The epidemiology of yellow fever in Arica. Microbes Infect 4: 1459-1468.

Paupy C, Brengues C, Damgang B, Herve JP, Fontenille D, Simard F 2008. Gene flow between domestic and sylvan populations of Aedes aegypti (Diptera: Culicidae) in North Cameroon. $J$ Med Entomol 45: 391-400.

Paupy C, Brengues C, Ndiatt O, Toty C, Herve JP, Simard F 2010. Morphological and genetic variability within Aedes aegypti in Niakhar, Senegal. Infect Genet Evol 10: 473-480.

Saunders EJ, Marfin AA, Tukei PM, Kuria G, Ademba G, Agata NN, Ouma JO, Cropp CB, Karabatsos N, Reiter P, Moore PS, Guber DJ 1998. First recorded outbreak of yellow fever in Kenya, 1992-1993. I. Epidemiologic investigations. Am J Trop Med Hyg 59: 644-649.

Smith CEG 1956. The history of dengue in tropical Asia and its probable relationship to the mosquito Aedes aegypti. J Trop Med Hyg 59: 3-11. 
Somers G, Brown JE, Barrera R, Powell JR 2011. Genetics and morphology of Aedes aegypti (Diptera: Culicidae) in septic tanks in Puerto Rico. J Med Entomol 48: 1095-1102.

Sylla M, Bosio C, Urdaneta-Marquez L, Ndiaye M, Black IV WC 2009. Gene flow, subspecies composition and dengue virus-2 susceptibility among Aedes aegypti collections in Senegal. PLoS Negl Trop Dis 3: e408.

Tabachnick WJ 1991. Evolutionary genetics and arthropod-borne disease: the yellow fever mosquito. American Entomologist 37: 14-26.

Tabachnick WJ 1993. Patterns of genetic variation and the ecology of the yellow fever mosquito, Aedes aegypti. In KC Kim, BA McPherson (eds.), Evolution of insect pests, John Wiley \& Sons, New York, p. 413-422.

Tabachnick WJ 2013. Nature, nurture and evolution of intra-species variation in mosquito competence for arboviruses. Int J Environ Res Public Health 10: 249-277.

Tabachnick WJ, Munstermann L, Powell JR 1979. Genetic distinctness of sympatric forms of Aedes aegypti. Evolution 33: 287-295.

Teesdale C 1955. Studies on the bionomics of Aedes aegypti (L.) in its natural habitats in a coastal region of Kenya. Bull Entomol Res 46: 711-742.

Trpis M 1972. Seasonal changes in the larval populations of Aedes aegypti in two biotopes in Dar es Salaam, Tanzania. Bull World Health Organ 47: 245-255.
Trpis M, Hausermann W 1978. Genetics of house-entering behavior in East African populations of Aedes aegypti (L.) (Diptera: Culicidae) and its relevance to speciation. Bull Entomol Res 68: 521-532.

Tsetsarkin KA, Chen R, Sherman MB, Weaver SC 2011. Chikungunya virus: evolution and genetic determinants of emergence. Curr Opin Virol 1: 310-317.

van Someren ECC, Heisch RB, Furlong M 1958. Observations on the behaviour of some mosquitoes of the Kenya coast. Bull Entomol Res 49: 643-660.

Vasilakis N, Cardosa J, Hanley KA, Holmes EC, Weaver SC 2011. Fever from forest: prospects for the continued emergence of sylvatic dengue virus and its impact on public health. Nat Rev Microbiol 9: $532-541$.

Verna TN, Munstermann LE 2011. Morphological variants of Aedes aegypti collected from the leeward island of Antigua. J Am Mosq Control Assoc 27: 308-311.

Wallis GP, Tabachnick WJ 1990. Genetic analysis of rock hole and domestic Aedes aegypti on the Caribbean island of Anguilla. J Am Mosq Control Assoc 6: 625-630.

Wallis GP, Tabachnick WJ, Powell JR 1983. Macrogeographic genetic variation in a human commensal: Aedes aegypti, the yellow fever mosquito. Genet Res 41: 241-258. 